\title{
THE LASER VIDEODISC IN THE FEDERAL REPUBLIC OF GERMANY
}

\author{
Ottmar Hertkorn
}

\author{
translated from German into English by \\ Dale V. Lally Jr., Editor-in-chief
}

\begin{abstract}
This article reports on research into the use of laser videodiscs for instruction in the Federal Republic of Germany (FRC). It describes the criteria for the selection of classroom media and explains four configurations of the videodisc. It also reports on the reactions of classroom teachers to the videodisc.

KEY WORDS: Videodisc, instructional technology, teacher training, foreign languages, interactive video.
\end{abstract}

\section{Introduction}

During the 1970's, an educational planning commission of the German federal government supported a project to develop a system of entry-level training as well as in-service teacher training via mediated instruction. Administered by the Center for Research and Development in Objective Teaching and Learning Methodology (Forschungs-und Entwicklungszentrum fur objektive Lehr-und Lernverfahren $\mathrm{GmbH}$ ) in Paderborn, a system was developed to enhance the training of incoming teachers as well as provide in-service updating for experienced teachers using mediated delivery systems.

One solution suggested for achieving this goal was to provide to teachers study materials for use in strategically placed learning carrels or workstations. One pre-requisite demanded that these study materials provide an adequate learning environment, located as close as possible to the teacher's home work location (in the hometown, media center, or school). In addition to the necessary hardware, these workstations must supply relevant theoretical literature, appropriate applied case studies and instructions for additional contacts.

Professor Ottmar Hertkom majored in Cermanistics at the University of Tubingen and holds the Doctorate in Literature from the State University of Sao Paulo, Brazil. For ten years he worked abroad in Brazil and Finnland for the German Academic Exchange Service and the Coethe Institute. He is currently Director of the Institut fur Unterrichtswissenschaft/Medieninformation, Postfach 15 67, 4790 Paderborn, Bundesrepublik Deutschland. 


\section{Videodisc Experiments}

Since 1977, following the successful development of media profiles, study guides, and workstations for such traditional modes of media delivery as transparencies, radio, and television, FEoLL has been conducting research on the effectiveness of the videodisc as a means of instruction. The first attempts were somewhat less than successful when technical obstacles prevented further use of the CED type videodisc originally offered by the Klett Publishing Co. for instructional use in the areas of biology, geography, and physics. However, since 1981, Klett Publishing has offered a laser videodisc for use exclusively in the field of biology.

\section{The Prime Criteria for Instructional Media-Learner Autonomy}

In order to identify appropriate criteria for emerging instructional delivery systems, the technology must be:

(1) user-friendly and designed for ease of operation.

(2) goal-oriented.

If one agrees that the new technology is being developed for the benefit of the learner and not for the teacher, then the following hypothesis is offered:

The quality of a medium is directly related to its capabilities for encouraging study.

In other words, regardless of the textual materials, the greater the possibilities for self-directed study, the greater is the teaching potential of the technology.

In the first century A.D., Quintilian observed that one cannot force students to be motivated. He did note, however, that external conditions, i.e., the learning environment, may either help or hinder the motivation to study. For this reason, one should select a medium which permits self-paced instruction or at least strives to approach that goal in a manner which is more effective than heretofore.

\section{Avoiding Technical Euphoria}

It is well known from extensive experience with teachers and students that not every technology-based delivery system is helpful to the learning process or to the student. For that reason, this new medium--the laser videodisc was tested to determine its suitability for use by students. It must be possible for either an individual or a small group to take full advantage of all the options offered by the system. This is a contrast to other delivery systems previously made available to schools in that now, for the first time, the student is given direct access to the textual materials. 


\section{Judging the Effectiveness of Mediated Delivery Systems}

Below are presented the most significant questions concerning the use of mediated delivery systems. These questions have traditionally been asked of each complex mode of mediated instruction including instructional video and computers.

1. Can I achieve my instructional goals more effectively via personal contact? If yes, then I do not need any technology or a mediated delivery system.

2. Can I also achieve my instructional goals via a simpler medium just as quickly and surely? If the answer is yes, then it should be possible to ignore video cassettes and use audio cassettes.

In regards to the laser videodisc, the questions should be:

1. How may I justify the use of this medium?

2. In what manner will my students derive a benefit from this medium: (Just saving time would constitute a tangible benefit)!

3. Would students derive more benefit without this medium?

4. If other means are available to achieve similar instructional goals, should I abandon this medium?

\section{Basic Videodisc Versions}

These are the major versions of the videodisc, based on the reflective optical principal:

1. film version

2. instructional version

3. programmed (automatic) version

4. individualized (unstructured) version

Versions 1 and 2 require a computer, while 3 and 4 do not. Of course there are intermediate versions, as, for example, between the film (1) and instructional (2) versions. When comparing the relative production costs of film vis-a-vis videodisc, from the simple linear programs to the programmable and individualized versions, videodisc production costs are vastly greater than film costs.

\section{The Film Version}

This version offers a linear, real time playback capability with no interruptions. It is suitable for entertainment films as well as demonstrations. Motion pictures or slide/tape shows may be shown and repeated as often as desired. (Each side of a videodisc will run up to 60 minutes.) However, when equipped with encoded frame numbers and a search mode, this version permits the viewer to start and stop the disc at any desired point. 


\section{The Instructional Version}

The instructional version features a "hands on" control of all player functions. Indeed, learners of all ages, from young children to adults, quickly master all of the control functions such as slow speed, stop, fast rewind and advance, and selection of one or both of the audio soundtracks. Since it is not possible to erase inadvertently either the picture or the soundtrack, this version gives to both the teacher and the learner the widest possible freedom of movement.

Each side of a videodisc may contain up to 79 segments, with each segment holding $\mathbf{5 7 0}$ individual frames. The viewer may select any one of the 54,000 individual frames on one side of the disc.

In contrast to the rigidly structured and programmed versions often encountered in computer-assisted instruction, the instructional version does not force the vierer into a lock-step mode, but rather encourages the user to choose his or her own path through the program. In my opinion, for the next few years, this version will offer the best possibilities for use in the school systems in the Federal Republic.

\section{The Programmed Version}

In the U.S., this version is appropriately called the "industrial" model and may be optimally used for in-house, industrial training. Detailed branching throughout the learning path is made possible through a built-in microprocessor which controls the starts, stops, and audio channels. Thus, multiple-choice questions, cloze procedures, variable program sequences, and self-testing are all possible. This version could be compared to an automatic dishwasher which offers several wash modes. Even though several different programs are available, each will run automatically in a pre-set manner once it has been selected. This is rightfully called the "automatic" or structured version.

\section{The Individualized Version}

This is the "intelligent" videodisc. It is interfaced to an external computer which controls endless variations in text, graphics, and symbol selection along with full control of all mechanical functions. This version naturally assumes that the user is familiar with the system operation and enjoys a certain amount of autonomy. At any time, the system may be switched back and forth between manual and automatic control. 


\section{Videodisc Qualities Which Are Important for Instructional Use}

The following videodisc qualities are important for academic utilization:

1. Video and tonal qualities are outstanding. The video quality, particularly of the still frames, is at least as good as any contemporary video system. The tonal quality is much better than any existing system. The video bandwidth is 5 megaherz, while the audio range extends from 40 cycles to 20 kilocycles.

2. The excellent visual and acoustical qualities remain totally unaffected, regardless of the length of time a frame is shown. This is due to the fact that nothing actually touches the disc. It can truly be said that a laser video disc is just as affected by the laser beam as a piece of paper is affected by the human eye.

3. The manufacturers maintain that the laser's expected service life is 5,000 hours. Because the encoded information is optically read, the videodisc remains unaffected by fingerprints or other surface impurities.

4. The videodisc format permits the user to search the disc in the same manner as a book. Obviously, one may compare videodiscs to books or other references works. The ability to search and recover the exact frame in a matter of seconds is as quick and simple as with a book or reference work. In contrast to video tape, changing videodisc players does not require the viewer to reset tape counters, since the frame numbers are imbedded in each frame. Furthermore, these frame numbers may be displayed or not, depending upon the viewer's choice. Finally, chapter numbers or film sequence numbers may be displayed in lieu of the frame numbers.

5. Since nothing comes into physical contact with the disc, each of the 54,000 frames may be read for as long as desired with no shimmering effect. In the still frame mode, however, there is no audio available. (Experiments with short duration sound tracks to accompany still frames are currently being carried out in the U.S.) Should the displayed frame or chapter numbers prove to be distracting, they may be deleted upon command.

6. Videodisc players are easy to operate. Even young learners are able to control the various functions, including random video sequence selection and rapid search mode, either directly on the player or through a remote control device.

7. Videodiscs offer dual sound tracks. Each video frame (motion pictures, slides, etc.) may offer supplementary 
textual materials, sound effects, or even music in two different versions. For instance, one soundtrack may be in English and the other in German. The same language may be offered on both tracks, but at differing levels of difficulty. In foreign language instruction, one audio channel could contain an instructional or didactic dialogue, while the remaining channel could present the original, authentically intoned language.

\section{The Lockstep Method and the Learning of Foreign Languages}

Obviously, pre-programmed methods can be utilized to teach foreign languages. Should one wish to learn a language such as Chinese, one should be willing to accept guidance and not resist the use of a rigid, structured, lock-step curriculum. However, the videodisc lends itself to either a lock-step or independent-study mode. In a poll distributed at a German university, both students and teachers overwhelmingly agreed that the videodisc will not result in a lock-step approach to instruction.

Should one want to see an overview of the various landscapes or cities, for example, a view of Germany from an aircraft, then one would prefer a method of randomly choosing the areas. This situation would not require any pre-programming. Thus, the instructional version, without an external computer, would suffice.

\section{More on the Use of Videodiscs to Teach Foreign Languages}

In preparing videodisc items for use in teaching foreign languages, one must seriously consider not only how videodiscs might be used to supplement natural dialogue situations, but also consider the situations where the use of a videodisc might be unnecessary. For example, in a purely cognitive area such as grammar instruction, it could be possible for the videodisc to offer benefits that in no manner compete with the teacher. One may justify the use of a videodisc in the classroom in order to relieve the instructor of the necessity for constant repetition (as with the Spanish language videodisc Montevidisco). The author feels that the total capacity of the teacher engaged in face-to-face instruction may be significantly enhanced through the use of machine-based simulated conversations.

If one assumes that free (unstructured) dialogue constitutes the most important objective for both teachers and students in the foreign language classroom, then the manually controlled instructional (\#2) version would be preferred over both the film (\#1) and automatic (\#3) versions. The individualized (\#4) version could be better utilized to present complicated instructional materials. 


\section{Experiences in the Federal Republic of Germany [FRG]}

According to WICAT, the U.S. government has ordered an instructional videodisc to teach biology. Using an experimental disc, students at three colleges required $30 \%$ less time to learn $20 \%$ more material than those students who did not have the videodisc.

As yet the German government has not officially mandated research on the use of the videodisc for instructional purposes. However, based on earlier experience with mediated information dissemination systems from the 1970's, FEoLL decided to provide selected biology teachers with a disc and a player to use in class for several weeks. The teachers would enjoy no benefits for participation (neither extra pay nor a reduced work load, as was common with projects in the 1970's).

\section{Classroom Experiences}

In 1982, 39 biology teachers, with 2139 students in 55 classes were given the videodisc Der Mensch (The Human Being) along with the necessary player. These teachers were located in secondary, comprehensive schools, and gymnasia. They recorded their experiences on a common questionnaire. In the following examples, the original questions are presented first, followed by the numbers and/or types of responses. In a few instances, the teachers' written comments are also included.

Question \#1: Analogous to the slide projector, could you comment on the still frame quality of the videodisc?

Responses: Of 39 responses, 36 teachers responded that they would use the videodisc.

Comments: "...one of the most sensible features of this device."

"...particularly valuable in the interpretation of figures (cerebral connections.)"

"...the still-frame feature replaced slides and transparencies. Thus there was a noticeable saving of time."

Additional note: Of 29 teachers, 23 indicated that the rapid review capability was used only for lesson preparation and not during class time.

Question \#2: $\quad$ Did you permit your students to operate the videodisc player, either singly, in pairs, or in a group?

Responses: Only six (6) teachers had permitted their students to operate the disc player.

Comments: This response corresponds to the views of other researchers seeking to increase learner autonomy, 
particularly through the use of media, namely, that the use of the videodisc remains too heavily dependent upon the teacher. There are, however, other arguments which seem to reinforce a hypothesis developed at the Heinrich Hertz Institute in Berlin in regards to televised instruction. It suggested that older learners who entertain serious doubts about the effectiveness of televised learning regard print media, mainly books, as their main source of information. On the other hand, young learners, and this includes the very next generation, eagerly spend relatively large amounts of time in front of a television set.

Even though, for various reasons, there was never any questionnaire submitted directly to the students, the next question/answer set provides an accurate indication of the teachers' views on the positive effects of the videodisc on the students' motivation.

Question \#3: Could you briefly describe the students' reaction (interest in, motivation, activities related) to the videodisc?

Response: Of a total of 36 teachers, 19 reported that the videodisc had a definite and positive impact on student motivation. The remaining 17 teachers indicated that the increased motivation which they had observed was a result of the fascination with the laser technology itself.

Question \#4: In your opinion, how could the videodisc be used for supplementary instruction?

Responses: $\quad$-use with headsets in the library

-for the preparation of experiments

-for the preparation of essays

Comments: Several instructors immediately pointed out that this new technology should be made available to the students after school. However, since most of the schools in the FRG are closed in the afternoon, "the current organization does not permit this." (Teachers and students normally have afternoons free.)

\section{Teaçhers' Opinion 1982: The Videodisc is the Medium of the Future}

On numerous occasions during in-service training, teachers from diverse academic areas were shown the identical videodiscs (Human Being 1: Brain/Stress and Sound Reception/Ear). Of 100 teachers polled, 87 clearly rejected the contention that the videodisc requires excessive preparation time. Even though those teachers were media users, they were certainly not utopian or overly enthusiastic. 
Fourteen of the teachers polled indicated that the videodisc represents the medium of the future.

It appears that print media are most widely accepted by teachers in the FRC. Using print media, the teacher remains in complete control with no fear of replacement by the book. Furthermore, if a book is used in conjunction with media, the teacher can always revert back to the book at will. This explains the agreement (93 out of 100) with the statement:

"The (main) advantage of the videodisc is the fact that I can stop its presentation at any time and inject my own explanations."

This certainly coincides with an earlier questionnaire distributed in the Frankfurt area in 1981 , whereby $96 \%$ of those teachers polled agreed with the statement:

"What I like most about the videodisc is the fact that I can start and stop the program whenever and wherever I desire."

\section{Teacher Reservations About Videodiscs}

Negative response to the videodisc was quite sparce. Understandably, three quarters of the responding teachers expressed concern about the lack of funds to purchase such equipment. Of 100 respondents, fully 50 indicated that the lack of funds will effectively prevent the acquisition of a videodisc system. While twenty-six disagreed, twenty-four remained undecided.

\section{Conclusion}

Even without any significant preparation, numerous teachers judged the use of the videodisc for instruction to be a "significant expansion of student autonomy."

In order to make all teachers aware of this medium, the optimal method is to offer teachers the instructional version using all of its advantages: ability to swiftly move from chapter to chapter, still frames, self-tests, proper documentation, suggestions for "things to $\mathrm{do}^{\prime \prime}$, and the choice of manual or automatic control.

\section{Notes}

1. The Klett Publishing Co., Stuttgart, developed a dual sided instructional videodisc entitled Der Mensch 1 [The Human Being 1]. One side is entitled Blick ins Gehirn [Looking into the Brain] and the reverse side is entitled Das menschliche Ohr [The Human Ear]. Intended for use in the 9th grade and above, it was edited by Bruno Oehring.

2. Stransfeld, Reinhard. Bildschirmtext und Building. Heinrich Hertz Institut fur Nachrichtentechnik. (Berlin: 1983).

3. The normal retail price of the basic videodisc player is $1298 \mathrm{D}$. Marks. Another configuration, featuring a built-in, programmable microprocessor and remote control capability, costs 1998 D. marks. 


\section{There's more to lab design... ...than meets the eyel}

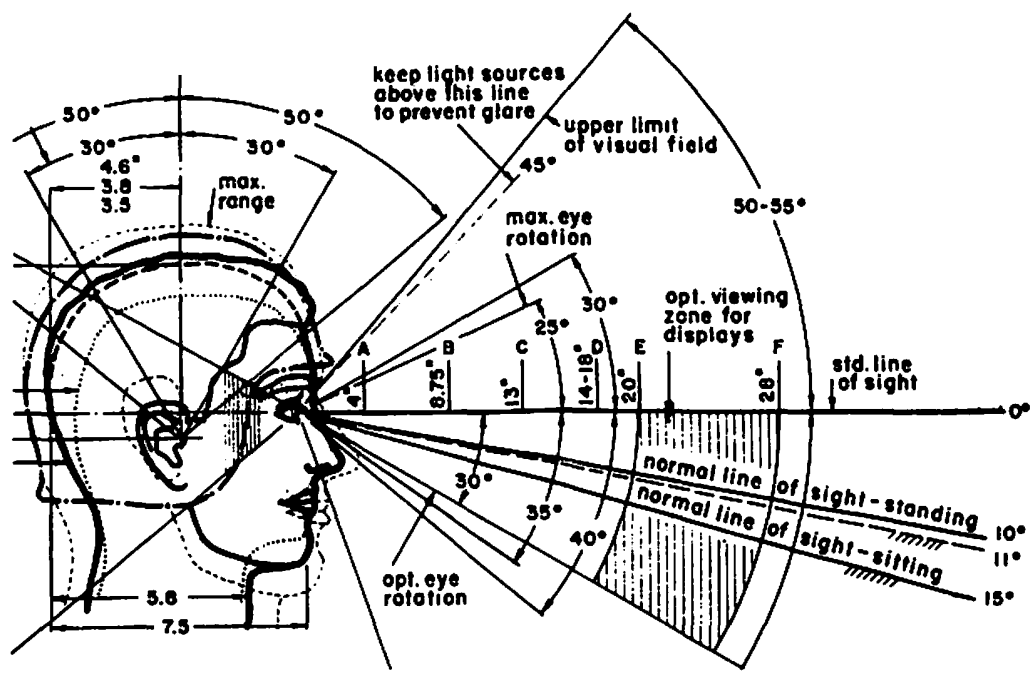

Sight lines. Head angles. Arm reach. And much, much more. Ignore these, and you jeopardize learning. Or, create a high-performance environment by building human factors principles into your lab design.

Which is our specialty. For eight years now we've been planning and installing turnkey learning centers in the US, Europe, \& the Middle East. They include language labs, micro-computer labs, multi-media LRC's, interactive video systems, and combinations of all four.

Planning. a new facility? Upgrading an existing one? Let us help. An informative packet of ideas and sample layouts is yours for the asking. Call (513) 561-4740 collect or write us at 7480 DeMar, Cincinnati, $\mathrm{OH} 45243$.

\section{Interactive Leaming Systems, Inc.}

Please mention the NALLD Journal when contacting our advertisers 